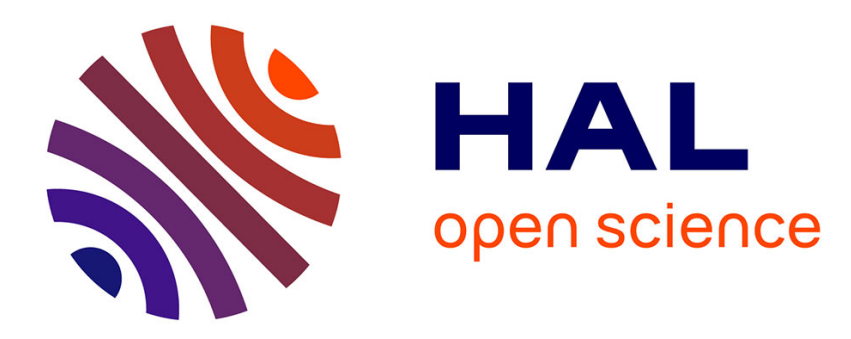

\title{
Sécurité informatique et valeur des écrits au travail Jérôme Denis
}

\section{To cite this version:}

Jérôme Denis. Sécurité informatique et valeur des écrits au travail. Semen - Revue de sémiolinguistique des textes et discours, 2009, 28, pp.85-100. hal-00437219

\section{HAL Id: hal-00437219 https://hal.science/hal-00437219}

Submitted on 30 Nov 2009

HAL is a multi-disciplinary open access archive for the deposit and dissemination of scientific research documents, whether they are published or not. The documents may come from teaching and research institutions in France or abroad, or from public or private research centers.
L'archive ouverte pluridisciplinaire HAL, est destinée au dépôt et à la diffusion de documents scientifiques de niveau recherche, publiés ou non, émanant des établissements d'enseignement et de recherche français ou étrangers, des laboratoires publics ou privés. 


\title{
Sécurité informatique et valeur des écrits au travail
}

\author{
Jérôme DENIS \\ LTCI (UMR5141) CNRS - TELECOM ParisTech \\ Département Sciences Économiques et Sociales \\ jerome.denis@telecom-paristech.fr \\ http://ses.telecom-paristech.fr/denis \\ www.scriptopolis.fr
}

2009. Semen $n^{\circ} 28$ (numéro spécial « Valeurs et enjeux des écrits au travail »), p. 85-100

\begin{abstract}
Résumé
Cet article interroge la valeur des écrits de travail non pas comme objet de recherche en soi, mais en tant que problème situé, discuté au sein même des organisations. II propose d'étudier le cas de la sécurité informatique en tant que registre d'attribution de certaines qualités aux écrits de travail, où la question de la valeur émerge en creux, définie face à des dangers. Les entretiens menés donnent à voir la difficulté de désigner ce type de valeur. Dans un premier temps, les personnes interrogées soulignent surtout l'absence de réelle sensibilité des documents électroniques qu'elles manipulent quotidiennement. Deux éléments sont mis en avant dans cette forme de disqualification: les liens indémêlables entre écrits et activité, et le régime de duplication généralisée dans lesquels ils sont pris. Trois domaines de valeur sont ensuite mis en lumière pour la sécurité informatique : la valeur stratégique, la valeur commerciale et la valeur juridique. À chacun correspond une manière spécifique d'appréhender les écrits au travail.
\end{abstract}

\section{Mots-Clefs}

Sécurité informatique ; écrits électroniques ; valeur ; pratiques d'écriture ; organisations

\section{Abstract}

This paper aims to question the value of writing at work as a situated problem, and not as a strictly research-driven object. It takes the case IT Security as a domain that assigns specific qualities to professional writings and within which value appears in relation with dangers. Interviews show how difficult such a value is to define. At first, people point a lack of real sensibility for the electronic documents they manipulate day to day. Two main elements are given: the entangled links between writings and action, and the general duplication regime within which they evolve. Then, three domains of value are enlightened: strategic, commercial and legal. For each of them, the paper shows there is a specific way to grasp writings at work.

\section{Keywords}

IT security; electronic writings; value; writing practices; organizations 
De très nombreuses recherches ont porté sur les pratiques d'écriture et de lecture électroniques au travail. Généralement, elles mettent en lumière les particularités des nouvelles technologies et les transformations qu'elles accompagnent à différents niveaux des activités professionnelles. Mais, inscrites dans le cadre élargi de la communication, elles traitent peu des liens qu'entretiennent ces pratiques avec le domaine de l'informatique dans les organisations. Inversement, les études qui portent sur l'informatique ne prennent pas en considération la nature scripturale des outils qu'elles conçoivent, implantent et réparent au fil du temps. Les deux faces de ces technologies semblent ne pas pouvoir être saisies ensemble, sans doute parce que la seconde relève d'un travail d'infrastructure que l'on peine à étudier dans toute son épaisseur. On sait pourtant, notamment depuis les travaux de S.L. Star (1998, 1999), l'importance de ce travail souterrain et le rôle qu'il joue dans la configuration des formes et des contenus eux-mêmes.

Le domaine de la sécurité informatique offre un site de recherche particulièrement riche pour opérer ce rapprochement. II donne une première entrée pour comprendre comment des outils et des objets d'écriture sont traités dans les termes de l'informatique. Aux yeux des ingénieurs responsables des systèmes d'information, les documents électroniques sont composés de données qui doivent être protégées de nombreux dangers : virus, perte, vol, altération... (Chateauraynaud \& Trabal 2007, Denis 2009b). Ce regard peut être déstabilisant pour les chercheurs qui étudient les pratiques d'écriture professionnelles, tant les catégories employées semblent éloignées de celles avec lesquelles ils ont l'habitude de travailler. On pourrait d'ailleurs adopter une démarche critique, qui consisterait à prendre ces catégories de haut et à disqualifier les manières dont on traite les écrits dans les entreprises au nom de la sécurité informatique pour montrer qu'elles passent à côté de tel ou tel aspect. L'objet de cet article est précisément de faire l'inverse : j'appréhenderai la sécurité informatique comme un monde spécifique d'existence pour les écrits au travail, au sein duquel ils sont dotés de certaines qualités. Un monde où, parce qu'il est question de savoir ce qu'il faut sécuriser et au nom de quoi, la question de la valeur est essentielle.

\section{Écrits au travail : en suivant quelques nouvelles pistes}

En France, notamment grâce aux travaux du réseau interdisciplinaire Langage et Travail, les pratiques d'écriture et de lecture au travail sont devenues un domaine de recherche à part entière. Depuis le début des années 1980, deux grandes directions ont été suivies pour l'investir. La première a consisté à articuler l'analyse de l'écrit aux problématiques qui soulignent les tensions entre " travail prescrit » et " travail réel ». II s'agissait de se dégager d'une vision qui restreindrait le domaine de l'écrit au seul monde de la prescription en soulignant l'importance des écrits du travail réel (Boutet 1993); mais aussi de questionner en profondeur les formes prescriptives de l'écrit (Charrasse 1992, Pène 1996). La seconde a consisté à documenter les relations étroites qui se nouent en situation de travail entre formes de communication écrites et orales (Grosjean \& Lacoste 1998, Pène 2001).

Depuis quelques années, on assiste à un renouveau des enquêtes sur l'écrit au travail. Celui-ci prend évidemment racines dans les questions soulevées par les premières études, mais il transforme aussi en partie la perspective de recherche elle-même. Sans entrer dans les détails, je retiendrai ici trois dimensions de cette transformation. La première est théorique : la notion de performativité, qui se trouve à la croisée de très nombreux intérêts de recherche (Denis 2006), est devenue un objet de réflexion essentiel, qu'elle soit utilisée comme outil d'analyse ou questionnée de front comme le fait $\mathrm{B}$. Fraenkel dans son programme de recherche sur les actes d'écriture (2006). Avec elle, c'est un véritable centrage pragmatique qui s'opère. L'écrit n'est plus seulement appréhendé comme un élément, certes important, d'un environnement de travail et d'action, mais comme une forme d'agir à part entière.

Ce renouvellement dans la manière de considérer les liens entre écriture et action nourrit directement le second déplacement qui s'opère dans les enquêtes récentes : on ne s'y 
intéresse plus seulement à la manipulation d'écrits en situation de travail, à leur mobilisation dans le cours d'action, mais aussi de plus en plus à leur fabrication. Le développement d'une pragmatique des écrits passe par la prise en compte de leur nature artefactuelle et donc une observation précise des conditions de production des objets graphiques dont on cherche à étudier la performativité. Cela s'est notamment traduit par un intérêt pour les professions de l'écrit, dans le domaine du droit (Fraenkel, et al. sous presses), de la science (Pontille à paraître) ou du travail administratif et commercial (Gardey 2008, Denis 2009a), renouant ainsi avec les travaux de sociologie des sciences et des techniques.

Enfin, avec l'informatisation et la mise en réseau généralisée, du web aux intranets en passant par le courrier électronique, les pratiques d'écriture ont subies de profondes transformations dans les quinze dernières années. Elles ont naturellement été prises en compte dans la plupart des enquêtes, certaines en faisant l'objet même de l'analyse (Licoppe 2002, Fraenkel \& Pontille 2003, Pène 2005), l'enjeu étant alors de saisir ce que l'électronique change dans le travail et dans sa communication.

Ces trois lignes rapidement esquissées ici dessinent autour des écrits au travail un territoire de recherche notablement modifié où les objets comme les questions ne sont plus tout à fait les mêmes. Cela est particulièrement sensible à propos de la notion de valeur. Avec le renforcement de la posture pragmatique, la place du chercheur a changé. Dans la plupart des premières enquêtes, la valeur des écrits était étroitement liée à celle des opérations d'écriture et de lecture. L'objectif était de souligner l'importance des formes langagières, communication orale comme pratiques d'écriture. II fallait montrer que ce monde des échanges langagiers était essentiel au travail et ne constituait pas un simple vernis de relations humaines (Lacoste 2001). Dans une perspective partagée avec l'ergonomie et la sociologie du travail traditionnelles, il fallait aussi mettre en lumière les compétences que tout cela supposait chez les opérateurs généralement considérés, y compris par eux-mêmes, comme étrangers à toute forme de litéracie (Charrasse 1992).

Dans tous les cas, il revenait au chercheur seul de désigner, une fois son enquête effectuée, la valeur des écrits au travail. Cela passait essentiellement par le vocabulaire des "fonctions" et la mise en avant de leur pluralité. C'est sur ce point que la posture pragmatique change beaucoup de choses. Avec elle, il faut accepter que les personnes que I'on observe traitent parfois elles-mêmes directement du problème de la valeur. Pour reprendre les termes de l'ethnométhodologie, la plupart des acteurs de l'on étudie développe leur propre théorie de la valeur.

L'objectif de cet article est d'investir une petite parcelle de ce vaste champ d'analyses qui saisit la valeur des écrits en tant que problème pour les personnes au travail, et non pas comme problème de recherche en soi. Pour cela, à condition que l'on accepte d'appréhender les flux de communication électronique dans leur dimension scripturale, le domaine de la sécurité informatique en entreprises est précieux puisqu'il est directement confronté à la question de l'évaluation de ce qu'il doit tenter de protéger. Je propose de l'étudier ici en tant que registre d'attribution de certaines qualités aux écrits de travail.

\section{Parenthèse méthodologique : l'entretien et le surgissement des doutes}

Cet article s'appuie sur une enquête menée par entretiens dans des organisations très différentes ${ }^{1}$. La sécurité informatique est un objet délicat à étudier, notamment parce qu'elle relève d'une forme d'évidence dont le chercheur a du mal à se saisir. Dans les entreprises, on ne trouve personne pour critiquer l'idée qu'il faille sécuriser les accès au réseau, les données électroniques et le matériel lui-même. La sécurité informatique est un bien en soi qui n'est jamais remis en question dans les entretiens. Elle représente ainsi la face sombre

\footnotetext{
${ }^{1}$ Recherche financée par le laboratoire SUSI de France Telecom R\&D, réalisée avec l'aide de Damien Guillaume. Les entretiens approfondis ont été effectués dans 43 entreprises (une heure trente en moyenne), avec 17 responsables informatiques et 26 utilisateurs finaux.
} 
de l'informatisation éclair du monde du travail dont beaucoup s'accorde dans un même temps à vanter le caractère inéluctable et à souligner la fragilité intrinsèque.

La méthode de l'entretien n'est pas facile à mettre en œuvre pour un tel objet, surtout si l'on attend d'elle qu'elle nous renseigne sur les "représentations" des personnes que l'on interroge, sur les « raisons » qui les poussent à agir, ou encore qu'elle nous donne accès à une série de faits présentés le plus objectivement possible. En revanche, si l'on assume qu'il est un moment de parole et de conversation parmi d'autres, on peut utiliser l'entretien à d'autres fin: non plus le focaliser sur les justifications les plus solides et les histoires les mieux construites, mais s'attarder sur les difficultés à s'exprimer et les doutes qui pointent. Dans cette perspective, c'est le processus de mise en mots qui compte, le travail qu'effectue la personne interrogée, non pas pour fouiller dans ce qui serait un stock de causes et de raisons emmagasinées quelque par dans son cerveau, mais pour produire un raisonnement en situation, en compagnie de l'enquêteur.

Or, durant plusieurs entretiens menés dans le cadre de cette enquête, un moment de doute assez radical est apparu. En tentant de définir la sensibilité des données qu'elles manipulaient au regard des risques informatiques qui avaient généralement été discutés auparavant, les personnes finissaient par remonter beaucoup plus en amont dans l'interrogation. Qu'est-ce que les documents qu'elles possédaient ou qu'on leur confiait avaient de véritablement sensible ? En quoi étaient-ils confidentiels ? Qu'est-ce que leur diffusion, ou leur perte, représenterait pour leur activité, celle de leur équipe, voire de leur entreprise ? Et les réponses à ces questions étaient surprenantes au regard de tous les arguments qui avaient été employés jusque là pour défendre ce qui était présenté comme les impératifs de la sécurité informatique : la plupart finissaient par dire que leurs données n'étaient " pas si sensibles que ça », autrement dit que les écrits électroniques de leur travail ne valaient pas qu'on prenne autant de précautions. Dans le pire des cas, leur disparition, leur dégradation ou leur divulgation, ne provoquerait pas de nuisances irréversibles.

Il faut bien sûr prendre au sérieux ce mouvement réflexif qui souligne plutôt la réussite de l'entretien que son échec. À propos de la sécurité informatique d'un point de vue général, il apporte d'abord quelques éléments de réponse à la flagrante absence de demande des usagers dans le domaine (Boullier, Jollivet \& Frédéric 2003). Mais surtout, il permet d'approcher la question de la valeur sous un angle original. On voit que celle-ci ne préexiste pas au travail des interviewés par lequel ils tentent en situation d'en faire émerger les contours. Dans le mouvement, deux pistes sont à suivre. Celle d'abord de l'affirmation que de très nombreux documents électroniques sont peu sensibles, voire sans valeur intrinsèque. II faut comprendre sur quels éléments s'appuie cette (dis)qualification. Mais la réflexion avançant, les personnes en viennent également à évoquer à propos de certains écrits qu'elles produisent ou manipulent ce qui pourrait être des critères de leur valeur dans les termes de la sécurité informatique. II faut aussi suivre ces réflexions afin d'en éclairer les fondements.

II va de soi que ce type d'exercice ne prétend pas à l'établissement d'une théorie générale sur la valeur des écrits au travail. Le but de cet article est au contraire de défendre l'idée que cette valeur n'est jamais isolable du registre dans lequel elle est définie. Ici, je ne prétends qu'étudier le cadre très spécifique de la sécurité informatique où la valeur est dessinée en creux, au miroir de la sensibilité de ce qu'il faut protéger. Plus généralement, il s'agit de souligner l'intérêt qu'il y a à interroger la valeur des écrits en tant que problème qui émerge, se stabilise ou au contraire fait débat dans des cadres très variés.

\section{Des écrits électroniques sans valeur?}

Lorsque les personnes interrogées affirment que leurs données ne méritent finalement pas l'attirail sécuritaire dont elles font l'objet, elles se réfèrent essentiellement à deux risques bien identifiés dans le domaine de la sécurité informatique : la perte et le vol. À leurs yeux donc, perdre certains écrits ou les voir entre les mains de la concurrence, ne constituent pas 
un drame irrémédiable. Comment expliquent-elles cela ? Deux arguments sont centraux : le premier souligne les liens entre écrits et activité, le second rappelle les opportunités que représente l'importante circulation et les incessantes duplications auxquelles oblige le travail collectif quotidien.

\section{Écrits situés et ancrage de la valeur}

Face à l'éventualité de pratiques d'espionnage ou de vol, les réactions sont nombreuses qui dénotent un doute fort sur l'existence même de telles pratiques dans le milieu des personnes côtoyées. Cette première dénégation passée, il fallait pour l'enquêteur forcer les choses pour pousser les logiques sécuritaires exposées dans leurs retranchements en invitant les personnes à imaginer des cas éventuels de fuites incontrôlées d'informations hébergées dans leurs outils informatiques, ou circulant sur le réseau entre postes à distance et serveur interne. C'est souvent dans le cadre de cette mise en situation forcée que la question de la faible sensibilité réelle des documents électroniques fait son apparition. L'argument principal qui permet au personne de faire ce type d'affirmation consiste à mettre en scène l'inutilité effective pour leurs concurrents de données qualifiées de "brutes ».

Ce faisant les personnes nient la validité d'une vision strictement informationnelle qui attribuerait aux données une valeur intrinsèque, désencastrée de toute dimension humaine et de tout travail interprétatif. On peut rapprocher cette manière de présenter les choses pour éloigner les craintes de vol ou d'espionnage des difficultés que rencontrent ceux et celles qui cherchent à mettre en place des processus de Knowledge Management. En soulignant à quel point ce qu'elles produisent (y compris à l'écrit et déjà stocké sur des disques durs) est sans valeur si on l'extirpe de son milieu, les personnes interrogées tendent un miroir à ces pratiques qui visent à " extraire » des connaissances avec les plus grandes difficultés, confrontées à l'extrême volatilité de ce qu'il s'agit de produire, stocker ou faire circuler (Rot 2005). Le paradoxe même du Knowledge Management réside dans cette violence faite à certains écrits du travail pour en fabriquer de nouveaux, épurés, censés pouvoir se passer de ceux qui les ont produits et se détacher des lieux et des moments de leur émergence.

Voilà ce que pointent les personnes dans cette enquête : les différentes données qu'elles transportent dans leurs ordinateurs portables ou font circuler par courrier électronique sont loin de constituer des "connaissances" prêtes à l'emploi. Les concurrents auraient beau s'en saisir, ils n'auraient pas à leur disposition l'expérience des situations, ni la connaissance interpersonnelle des clients, ni les savoirs accumulés nécessaires à leur compréhension.

On ne crée pas comme ça de toutes pièces une activité comme la nôtre. Ce n'est pas en termes capitalistiques, c'est plutôt en termes de compétences et d'expérience, d'expertise. Imaginons - ce serait quand même un problème -, que quelqu'un vienne pomper tout ce qu'on a sur notre serveur, ça ne suffit pas, puisque pour l'exploiter, il faut avoir les capacités à l'exploiter. (Directeur général, SSII Conseil).

Sur le versant de la confidentialité, l'absence de véritable sensibilité est donc expliquée par l'entrelacement fort de la plupart des écrits informatisés et de l'activité qui les contextualise et les historicise. C'est de ce lien qu'ils tiennent leur valeur et c'est ce qui fait que celle-ci n'est en définitive pas susceptible d'être « volée » ou « espionnée ».

\section{Un régime de duplication généralisée}

Une autre dimension émise par les personnes interrogées, non plus à propos du vol et de l'espionnage, mais de la perte. Les politiques de sécurité informatique en entreprise visent en effet aussi à prévenir des mauvaises manipulations, défauts de sauvegarde et autres pannes matérielles qui pourraient donner lieu à une disparition soudaine des documents informatiques qui font le quotidien du travail. Face à ce risque, les réflexions au cours des entretiens sont du même ordre que précédemment: les conséquences de la perte ou de l'inaccessibilité des écrits électroniques ne sont pas considérées comme dramatiques. 
Le raisonnement consiste ici à mettre en doute l'idée de disparition elle-même. Dans la plupart des cas, ça n'est pas parce qu'un texte, un document de travail, ou un message électronique ne sont plus accessibles sur les supports dont on dispose personnellement (disque dur, support externe, espace de stockage en réseau), qu'ils sont effectivement perdus. Pourquoi ? Parce que ces écrits sont très rarement uniques, qu'on peut les retrouver ailleurs sous une forme quasiment identiques à celle que l'on a perdue.

Mon collègue Suisse a perdu des trucs parce que son disque dur a planté, il n'avait pas fait de back-up. J'étais embêté, parce que j'en ai subi les conséquences aussi. On en subit les conséquences aussi. Mais les conséquences ne sont pas si lourdes. [...] . On se débrouille toujours, dans les équipes... II y a toujours quelqu'un qui a la sauvegarde du mail de l'autre. II y a toujours... On a toujours les données, d'autres versions. Donc c'est... La perte de productivité est faible, en fait. Elle n'est jamais considérable. Elle est lourde pour l'utilisateur, mais pour le projet collectif, elle est quand même raisonnable. (Responsable technique, Édition universitaire Web)

Contrairement à la situation précédente, l'argument défendu ici ne met pas en débat la portée informationnelle des écrits électroniques ou la nature des données informatiques. II n'est pas non plus tourné vers des fonctionnalités qui seraient purement techniques. C'est le milieu dans lequel évoluent ces données qui est considéré comme porteurs de qualités spécifiques qui déplacent la valeur des écrits.

Depuis quelques années, les conditions d'accomplissement du travail se sont transformées, inversant complètement le rapport à la communication. De pratique ignorée, voire en partie sanctionnée, elle est devenue objet d'une véritable injonction, dans un monde organisé en réseaux nécessitant un travail relationnel intensif (Boltanski \& Chiapello 1999, Nardi \& Whittaker 2002). L'importance que prennent aujourd'hui les formes de coopération dans le travail, associée à l'essor des technologies de circulation des informations sont ici pointés comme les conditions naturelles d'un régime de la duplication généralisée qui assure une forme de pérennité distribuée à la grande majorité des écrits de travail. Les échanges entre collègues, avec la hiérarchie, les prestataires ou les clients installent de fait une quasiimpossibilité de véritablement perdre quoi que ce soit. De ce point de vue, un exemplaire, figurant sur tel support informatique n'a pas de valeur en soi, si ce n'est celle de la facilité d'accès. Inversement, la reproductibilité et la circulation massive des écrits électroniques leur donnent une certaine valeur à l'échelle du travail quotidien : une présence et une permanence distribuée.

\section{Quelques contours de ce qui vaut d'être sécurisé}

Si les deux arguments précédents doivent être entendus et pris au sérieux, ils ne doivent pas être compris comme une manière pour les personnes de court-circuiter définitivement une évidence sécuritaire qui reste incontournable à leurs yeux. Du point de vue des politiques de sécurité informatique, ils soulignent plutôt l'enjeu essentiel de l'évaluation et de la qualification des données car si le doute sur la sensibilité des données et la gravité de leur vol ou de leur perte est largement partagé, il ne concerne pas tous les écrits professionnels. À la périphérie de la plupart des documents concernés se trouvent des écrits qui semblent avoir une forte valeur au regard des risques contre lesquels luttent les dispositifs de sécurité informatique. Sans prétendre à l'exhaustivité, l'enquête présentée ici permet de repérer quelques domaines de valeur : la stratégie et ses exigences de confidentialité, le marché et les écrits qu'on y vend, le droit et les restrictions de circulation qu'il impose.

\section{Documents stratégiques et secret}

Les écrits qui sont le plus facilement qualifiés de sensibles face aux risques informatiques sont ceux que les personnes qualifient de stratégiques. Ces documents sont présentés comme des éléments importants de la marche de l'entreprise. D'un point de vue de sociologie du travail, ils accompagnent ce que A. Strauss appelle le "processus 
d'organisation" (1988) en offrant des points d'appui essentiels pour faire tenir ensemble différentes lignes de travail. Ils enregistrent et établissent des décisions majeures, ils détaillent les différents éléments des projets... Mais dans le registre de la sécurité informatique, ça n'est pas de ce point de vue intra-organisationnel que leur valeur est identifiée. Ces écrits ne sont jugés sensibles qu'au regard de ce qu'ils pourraient représenter s'ils tombaient entre les mains des concurrents. C'est d'ailleurs dans ce sens qu'il faut prendre le qualificatif flou de stratégique : une information stratégique est une information qui, si elle tombait entre les mains des concurrents, leur donnerait à voir les plans et les projets que l'entreprise se donne pour son développement, ce qui lui ferait perdre son avantage dans le secteur.

Pendant un cycle de vente, on est souvent en concurrence avec deux ou trois autres concurrents. Donc, c'est un peu un trio, un podium là et on ne sait qui c'est qui va être le numéro un quoi. Des fois, ça dure trois semaines, des fois ça peut durer 6 mois. Donc là, il se passe des choses. II y a des entretiens. Donc chacun essaie d'attaquer, contre-attaquer. Donc là, si vous obtenez pendant ces trois ou 6 mois, une information effectivement sur le concurrent, qui peut jouer en votre faveur... Ben, il est clair que vous allez la mettre en avant. Et nous des fois, effectivement on a des choses qui apparaissent en interne, qu'on va bien effectivement éviter de divulguer (Responsable clientèle, Services Web).

Cette exigence de confidentialité vaut aussi à l'intérieur même des entreprises, pour des documents informatiques qui ne concernent plus des stratégies techniques ou commerciales, mais l'organisation elle-même. C'est essentiellement le cas des très nombreux écrits du domaine des ressources humaines. Ces derniers sont considérés comme tout aussi sensibles puisqu'ils donnent à voir des mécanismes décisionnels de l'action managériale (montant des primes, augmentations, projets de licenciement, etc.) et sont susceptibles de constituer pour les syndicats par exemple des moyens de pression ou de négociation dont on préfère qu'ils soient dépourvus.

Un exemple typique : en ce moment, on est en train de négocier les augmentations de salaire. Donc ça, tant que c'est pas fixé enfin, tant que c'est pas signé, c'est pas fait. Et puis, en plus ils ont pas... Moi j'ai les informations qui concernent la totalité de l'équipe. Ou même la stratégie d'augmentation, etc. Donc, ils ont pas forcément à être au courant de toutes ces choses là. (Directrice régionale, laboratoire pharmaceutique).

À l'inverse des documents évoqués plus haut qui sont considérés comme sans valeur dès lors qu'ils circulent hors de leur environnement direct d'usage, il existe donc des écrits dont la valeur est définie par rapport à leur fuite redoutée et ses conséquences potentielles. En terme de politique sécuritaire, ce type de documents est l'objet de traitements spécifiques plus ou moins radicaux selon les cas. Certains sont par exemple interdits de toute mise en réseau, stockés sur des disques durs off line auxquels un nombre très limité de personnes peut accéder. D'autres sont plus simplement présents dans des espaces de stockage régis par des systèmes de cryptage et de droits d'accès restreints.

De quoi est faite la valeur qui est mise en avant par les personnes qui présentent ce type d'écrits professionnels comme sensibles? Deux choses semblent importantes pour la caractériser. Tout d'abord, les écrits électroniques ne sont pas considérés ici en tant qu'objets ou documents, mais comme porteurs d'informations. Ils sont traités comme des supports qu'il faut manipuler avec précaution afin de préserver un contenu qui est considéré comme relativement indépendant.

Par ailleurs, cet état n'est pas permanent. La valeur de ces écrits électroniques est affaire de cadrage temporel. Les éléments stratégiques ne le sont que tant qu'ils désignent des options futures, des pistes qui seront suivies dans l'entreprise, généralement à court ou moyen terme. Dès que les décisions sont mises en œuvre et donc rendues publiques, les documents qui ont aidé à leur élaboration changent de statut, désormais destinés aux 
archives ou au rebut, selon les cas. La valeur se mesure donc ici en " coups d'avance " dans des processus de compétition ou de négociation.

\section{Écrits à vendre}

Parmi les écrits électroniques que l'on cherche à protéger à tout prix, on trouve d'autres éléments liés au marché qui n'ont plus rien à voir avec les processus décisionnels. Ce ne sont plus des équipements de l'activité organisationnelle, mais des produits en tant que tel. Ils sont évidemment pléthore dans le secteur de l'innovation technologique où ce ne sont jamais seulement des objets manufacturés qui circulent, mais du code informatique, des procédés, etc. Ces écrits de l'innovation sont rares et considérés comme le cœur de ce qui doit être protégé.

Après, dans cette boîte [le décodeur audiovisuel], il y a le software. C'est du code qui est développé en interne par des ingénieurs chez nous, qui va regrouper l'intelligence de la boîte, c'est-à-dire tous les services que vous allez être capable d'offrir avec le produit que vous êtes en train de construire. Ça, c'est relativement sensible, parce que ce n'est que des choses qui sont développées en interne. Et là, il y a un véritable risque. (DSI, SSIl et audiovisuel).

On se trouve dans une situation opposée à celle qui amenait les personnes à insister sur la dimension contextualisée de leurs écrits électroniques et à souligner leur ancrage immuable dans l'activité. Les écrits que l'on protège ici ont été fabriqués pour circuler. C'est un processus de désencastrement progressif qui les a constitué en " biens " qui peuvent être mis en marché (Callon, Meadel \& Rabeharisoa 2000). Leur valeur tient précisément à cette capacité à passer d'un fournisseur à ses clients, détachés des situations locales de leur production.

Celle-ci est directement exprimée en termes économiques par les personnes qui les évoquent. Alors que la plupart des évaluations pessimistes qui cherchent à mesurer les pertes que représenterait l'éventualité d'une panne d'un disque dur s'expriment en temps de désagrément, celles qui envisagent la perte d'écrits de ce type s'appuient sur un prix clairement établi.

Moi, je travaille sur des modèles [économiques dans le domaine du transport]. Alors, je sais pas si vous imaginez, mais fabriquer un modèle comme ça coûte pas 300 euros. Ça coûte quelques milliers d'euros. II y a même des modèles plus chers sur des grandes villes qui coûtent des dizaines de milliers d'euros. Donc, il faut se rendre compte que... c'est sûr que si on en paume un... Bon on va toujours avoir une vieille version quelque part... On s'en sortira. Mais, tout le travail qu'il faudra recommencer ça peut être assez cher. (Ingénieur conseil libéral).

En terme de sécurité informatique, cela veut dire que la sensibilité de ces écrits n'est pas seulement attachée à la question de la confidentialité et de l'espionnage, mais qu'elle renvoie également aux risques de perte et de dégradation. Leur stockage et leur sauvegarde récurrente sont aussi importants que leur protection contre le vol. Leur disparition pourrait directement menacer l'existence même de l'entreprise dont ils constituent le patrimoine.

\section{Les valeurs du droit}

Le droit tient une place importante dans l'exploration que mènent les personnes en situation d'entretien pour cerner la valeur des écrits électroniques. II est d'abord évoqué comme un registre spécifique de qualification qui désigne certains types de documents comme devant être protégés. En entreprise, c'est le cas essentiellement des "données personnelles ». L'enquête ne permet pas d'entrer dans les détails de ce vaste monde qui, du point de vue des écrits au travail, mériterait une recherche à lui tout seul. Retenons simplement que la sécurité informatique en entreprises est en partie imposée par le droit qui exige que tel ou tel type de données bénéficient d'une confidentialité maximale. On retrouve ici les mêmes enjeux que dans le domaine des documents stratégiques: c'est en tant que supports 
d'informations considérées comme sensibles que ces écrits, relevant généralement des ressources humaines, sont traités.

Mais le droit comme espace de mise en valeur des écrits de travail est également évoqué sous un angle très différent. II n'y apparaît plus en tant que prescripteur de sécurité mais au contraire comme instance de risques. C'est un droit que l'on redoute parce qu'il pourrait se mettre à se saisir de certains documents qui permettraient d'établir l'existence de pratiques discutables, voire clairement illégales.

Évidemment cet argument n'est pas présenté de front en entretien. II faut pour l'évoquer en passer par des histoires médiatiques ou en appeler aux pratiques d'autres que soi. Mais le risque est bien là et les conséquences de la fuite de ce genre d'écrits (qu'elle soit accidentelle ou orchestrée par la concurrence) seraient catastrophiques.

Je travaille avec la grande distribution [...] oui, très clairement, l'information est très très sensible. D'autant plus sensible, qu'on travaille aujourd'hui avec des enseignes qui ont des contraintes très claires, notamment vis-à-vis de la loi Dutreil, où on oblige les enseignes à être très transparentes sur les niveaux de marges, sur les niveaux de facturation, sur les prix de revente à perte. Donc toutes ces informations qui sont parfaitement légales, même si tous les acteurs ne font pas forcément tout très proprement, on se doit, tout du moins en façade, de l'être. Certaines des informations que je possède sur mon ordinateur aujourd'hui possèdent un caractère ultra confidentiel...et il serait très préjudiciable, pour ma société, tout comme pour moi, si ces informations venaient à tomber dans les mains de la concurrence. (Commercial, distribution).

Cette valeur liée au droit est évidemment très difficile à appréhender et ne peut faire l'objet d'une désignation frontale. On peut cependant faire l'hypothèse qu'elle pèse de manière importante dans certains secteurs et que la frontière qui la sépare du caractère stratégique de certaines informations n'est pas complètement étanche. Par définition, les documents concernés sont dans la plupart des cas difficiles à désigner a priori, à moins d'en assumer explicitement le caractère illégal. Si dans l'extrait précédent, il semble assez clair que la personne dispose de documents directement compromettant, comment trancher à propos de certains courriers électroniques par exemple?

C'est que la valeur dont il est question renvoie à un mode d'existence pour les écrits au travail complètement différents de ceux que nous avons croisés jusque là. Les documents sont appréhendés ici en projection, en tant que preuves potentielles. Le risque pèse sur eux de participer à une traçabilité à rebours, très éloignée des dispositifs de traçabilité contrôlée qui sont mis en place depuis quelques années dans les entreprises. Les traces seraient ici fortuites. La valeur de preuve ne peut être attribuée que plus tard, dans des circonstances qui transformeront largement le regard que l'on aura sur les écrits en question, qui seront alors traités comme des indices (Ginzburg 1980). Dans le monde de la sécurité informatique se développe donc, parallèlement à l'attention que l'on porte à des documents assez clairement identifiés comme sensibles, une inquiétude plus ou moins grande, une "heuristique de la suspicion" (Brown \& Lightfoot 2002), à propos de pratiques d'écriture électronique dont les frontières sont floues et mouvantes.

\section{Conclusions}

La sécurité informatique est une préoccupation partagée dans les organisations, à laquelle sont associés un vocabulaire, des pratiques et des dispositifs techniques. Elle constitue un registre de valeurs spécifique pour les écrits de travail. Parce qu'elle les donne à voir en creux, définies face à des dangers, elle facilite au chercheur la mise à distance du regard trop souvent fonctionnaliste qui est porté sur les nouvelles technologies lorsqu'il s'agit de pratiques d'écriture.

L'entrée par le doute sur la sensibilité des écrits électroniques et l'exploration qui le suit au cours des entretiens nous a permis de repérer les principaux territoires de la valeur 
sécuritaire. Leur étroitesse d'abord : la plupart des documents manipulés, reçus, envoyés, ne valent pas aux yeux de leurs lecteurs ou auteurs l'arsenal de précautions qu'on voudrait bien leur prêter pour empêcher qu'ils soient volés ou perdus. Deux éléments fondent ce diagnostic : la plupart des écrits électroniques ne sont riches que de leur ancrage dans l'activité ; et leur présence quasi-permanente est assurée par la duplication et la circulation qu'exigent aujourd'hui les organisations dites flexibles. Mais l'enquête a aussi fait émerger trois formes de valeurs pour les écrits électroniques au travail: la valeur stratégique, la valeur commerciale et la valeur juridique. À chaque fois, les écrits sont saisis différemment. Dans le premier cas, ils sont traités comme les supports fragiles d'informations confidentielles; dans le deuxième ils sont élevés au rang de biens aux qualités marchandes stabilisées ; dans le troisième, on en appréhende la capacité à faire preuve ultérieurement.

À travers cet exemple, j'espère avoir montré l'intérêt qu'il y a à adopter une posture ouverte à la variété des définitions de la valeur des écrits. Celle-ci n'est jamais donnée, ni clairement établie. Dans les entreprises, la désignation des pratiques d'écriture ou des types de documents qui "comptent" est un problème complexe qui, précisément parce qu'il est pris dans des registres très différents, est rarement stabilisé. Dans de nombreux secteurs d'activité, la production et la manipulation d'écrits électroniques sont soumises à des exigences de productivité et de qualité, déjà difficiles à articuler (Denis 2009a) auxquelles les préoccupations sécuritaires viennent s'ajouter.

Mais porter attention à la manière dont s'agencent ces registres en situation ou, comme c'est le cas ici, explorer les limites de l'un d'eux, n'est pas seulement un moyen de mettre au jour des logiques différentes. On l'a vu, une valeur s'exprime toujours attachée à une certaine définition de l'écrit, de sa forme, de son objet, comme de sa pratique. Parce qu'elle met en lumière les qualités variables des entités graphiques qui circulent dans les entreprises et qu'elle en laisse la définition à charge de ceux qu'elle observe, c'est aussi sur les ontologies situées des écrits au travail que cette posture de recherche peut informer.

\section{Bibliographie}

Boltanski, L. \& Chiapello, È. 1999, Le nouvel esprit du capitalisme, Gallimard, Paris.

Boullier, D., Jollivet, P. \& Frédéric, A. 2003, 'L'institution de la sécurité ou comment s'en désintéresser', Les Cahiers du Numérique, no. 3-4, pp. 219-39.

Boutet, J. 1993, 'Écrits au travail', in B Fraenkel (ed.), Illétrismes. Variations historiques et anthropologiques, BPI-Centre Georges Pompidou, Paris, pp. 253-66.

Bowker, G. C. \& Star, S. L. 1998, 'Building Information Infrastructures for Social Worlds. The Role of Classifications and Standards', in T Ishida (ed.), Community Computing and Support Systems, , Springer, Berlin, pp. 231-48.

Brown, S. D. \& Lightfoot, G. 2002, 'Presence, Absence, and Accountability: E-mail and the Mediation of Organizational Memory', in S Woolgar (ed.), Virtual Society? Technology, Cyberbole, Reality, Oxford University Press, Oxford, pp. 210-29.

Callon, M., Meadel, C. \& Rabeharisoa, V. 2000, 'L'économie des qualités', Politix, vol. 13, no. 52, pp. 211-39.

Charrasse, D. 1992, 'L'usine, l'écriture et la place', Genèses, no. 7, pp. 63-93.

Chateauraynaud, F. \& Trabal, P. 2007, 'Des vigiles invisibles. Les administrateurs-réseaux et la sécurité informatique', Annales des Télécommunications, vol. 62, no. 11-12.

Denis, J. 2006, 'Les nouveaux visages de la performativité', Études de communication, no. 29, pp. 7-24. 
---- 2009a, 'Le travail invisible de l'information', in C Licoppe (ed.), L'évolution des cultures numériques, de la mutation du lien social à l'organisation du travail, FYP, Paris, pp. 117-23.

---- 2009b, 'Les ressorts de la sécurité informatique. Des hommes, des machines et des données', in C Licoppe (ed.), L'évolution des cultures numériques, de la mutation du lien social à l'organisation du travail, FYP, Paris, pp. 190-9.

Fraenkel, B. 2006, 'Actes écrits, actes oraux : la performativité à l'épreuve de l'écriture', Études de communication, no. 29, pp. 69-93.

Fraenkel, B. \& Pontille, D. 2003, 'L'écrit juridique à l'épreuve de la signature électronique, une approche pragmatique', Langage et Société, no. 104, pp. 83-121.

Fraenkel, B., Pontille, D., Collard, D. \& Deharo, G. Sous presses, Le Travail des huissiers : transformations d'un métier de l'écrit, Travail et activité humaine, Octares, Toulouse.

Gardey, D. 2008, Écrire, calculer, classer. Comment une révolution de papier a transformé les sociétés contemporaines (1800-1940), La Découverte, Paris.

Ginzburg, C. 1980, 'Signes, traces, pistes. Racines d'un paradigme de l'indice', Le Débat, no. 6, pp. 3-44.

Grosjean, M. \& Lacoste, M. 1998, 'L'oral et l'écrit dans les communications de travail ou les illusions du "tout écrit"', Sociologie du travail, vol. XL, no. 4, pp. 439-65.

Lacoste, M. 2001, 'Peut-on travailler sans communiquer ?', in A Borzeix \& B Fraenkel (eds), Langage et travail : communication, cognition, action, CNRS éditions, Paris, pp. 21-53.

Licoppe, C. 2002, 'Le traitement des courriers électroniques dans les centres d'appels', Sociologie du travail, vol. 44, no. 3, pp. 381-400.

Nardi, B. A. \& Whittaker, S. 2002, 'NetWORKers and their Activity in Intensional Networks', CSCW, vol. 11, no. 1-2, pp. 205-42.

Pène, S. 1996, 'La procédure entre contrainte et subjectivité. Approche linguistique', Sciences de la Société, no. 30, pp. 53-67.

---- 2001, 'Les agencements langagiers de la Qualité', in A Borzeix \& B Fraenkel (eds), Langage et travail : communication, cognition, action, CNRS éditions, Paris, pp. 303-21.

---- 2005, 'La vie des "hommes infâmes" dans la société de disponibilité', Études de communication, no. 28, pp. 107-24.

Pontille, D. à paraître, 'Updating a Biomedical Database: Writing, Reading and Invisible Contribution'. in D. Barton \& Uta Papen (eds) Anthropology of Writing: Understanding Textually-Mediated Worlds. London: Continuum

Rot, G. 2005, 'Le Knowledge Management et l'économie spontanée du partage des connaissances. Propos sur un desinvestissement de forme', Économies et Sociétés, vol. 25, no. 4, pp. 675-8.

Star, S. L. 1999, 'The ethnography of infrasctructure', American Behavioural Scientist, vol. 43, no. 3, pp. 377-91.

Strauss, A. 1988, 'The articulation of project work: an organizational process', Sociological Quarterlly, vol. 29, no. 2, pp. 163-78. 